Alma Mater Studiorum - Università di Bologna DEPARTMENT OF ECONOMICS

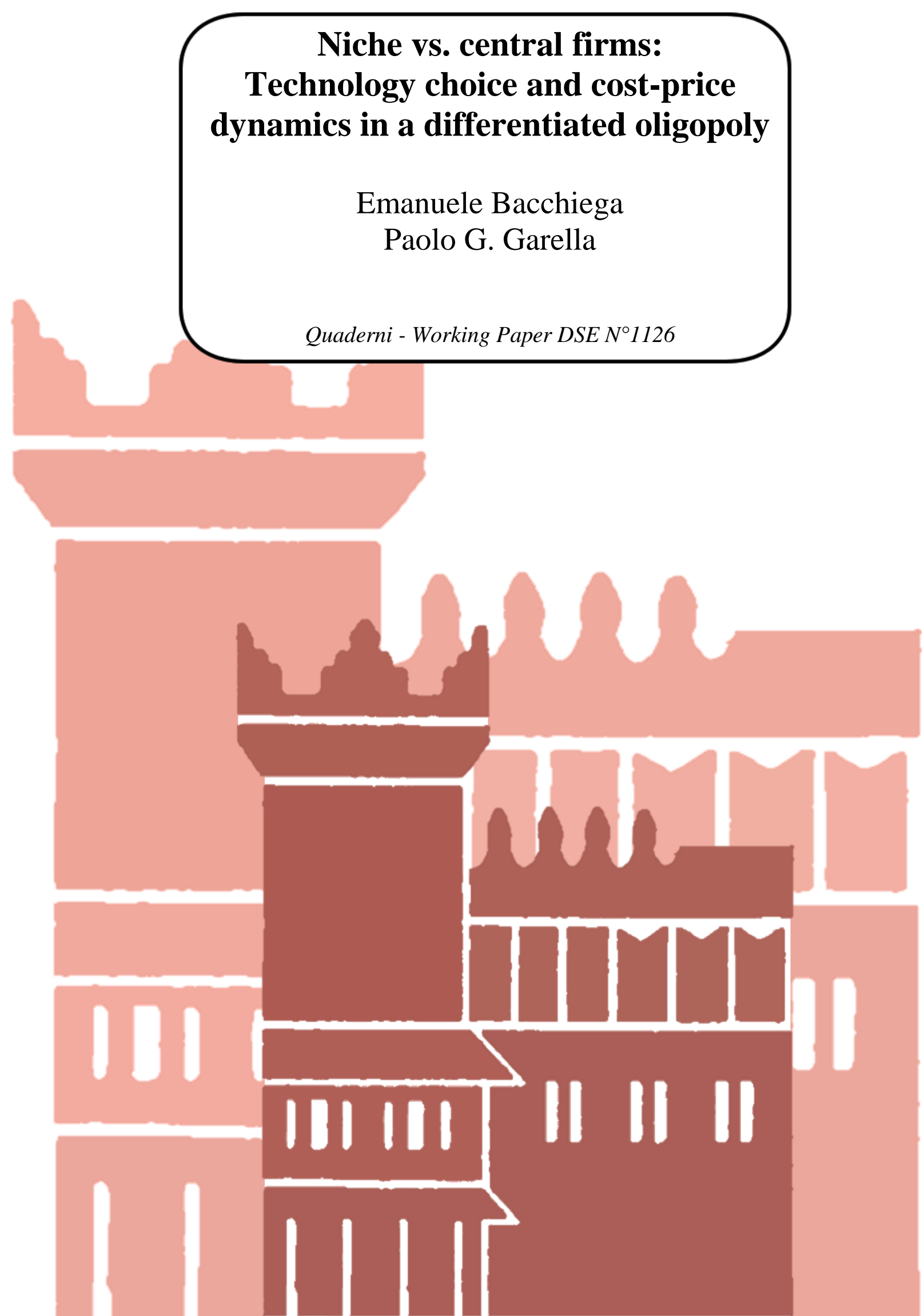




\title{
Niche vs. central firms: Technology choice and cost-price dynamics in a differentiated oligopoly
}

\author{
Emanuele Bacchiega*1 and Paolo G. Garella ${ }^{\dagger 2}$ \\ ${ }^{1}$ Dipartimento di Scienze Economiche, Alma Mater Studiorum - Università di Bologna, Italy. \\ ${ }^{2}$ Dipartimento di Economia, Management e Metodi Quantitativi, Università di Milano, Italy.
}

October 12, 2018

\begin{abstract}
This paper is about technology choices in a differentiated oligopoly. The main questions are: whether the position in the product space affects the choice of technology, how changes in fixed costs affect price outcomes, the strategic responses to policy interventions. The industry is an oligopoly where a central firm is competing with two peripheral (or marginal) ones. The former is shown to be more ready than the latter to adopt a technology with low marginal costs and high fixed costs (Increasing Returns to Scale) rather than one with the opposite pattern (Constant Returns to Scale). The fixed cost in the IRS affects the technology configuration and hence output prices. For instance, a lower fixed cost may trigger lower prices and it is neutral only for given technologies. A price-cap may forestall a change in technologies; nondiscriminatory ad-valorem tax and taxes on variable input, or discriminatory unit taxes can also affect the technology pattern and deliver important effects on prices.
\end{abstract}

Keywords: Oligopoly, technology, price dynamics, policy intervention.

JEL classification: D43, L11, L13.

${ }^{*}$ 凶emanuele.bacchiega@unibo.it

${ }^{\dagger}$ 凶paolo.garella@unimi.it 


\section{Non-technical summary}

In a wide range of industries, competing firms adopt different technologies. Two explanations of this penomenon have been prompted by the economic literature. On the one hand, technological asymmetries are related to the evolutive process of technological diffusion, on the other hand they are a direct consequence of the strategic behavior of firms. In this article we take the second stance and analyze how firms select their productive technology when they produce differentiated products. In particular, we are interested in (i) unveiling the linkages between the location of firms in the product space and their technological choices, (ii) analyzing the effects of these choices on the price level in the industry and (iii) assessing the effects of some commonly implemented policy instruments in this setup. Our results (i) describe how changes in the characteristics of the technologies affect the choices of firms, (ii) show that these choices are consequential regarding the price level in the industry and (iii) suggest that the possibility to modify the operating technology may increase or decrease the effectiveness of commonly used policy instruments. 


\section{Introduction}

In a wide range of industries competing firms adopt different technologies. One reason is that firms discover and adopt new technologies at different timing, as empirically documented as early as Griliches (1957); Dunne (1994); Doms et al. (1995). This heterogeneity has received various explanations. Two main branches in the economics literature can be identified: ${ }^{1}$ one is related to the diffusion of new technologies; in a pure evolutionary view, asymmetric choices are "transitory" from the single firm viewpoint but permanent in the evolution of an economic system (e.g. Dosi, 1997 and the literature cited therein); in Growth Theory the main contribution comes from "vintage capital" models originated from the work of Johansen (1959) (most related to the issue is Jovanovic (1998); see also Boucekkine et al. (2011) for a survey). A second branch, to which this paper refers, takes an Industrial Organization approach. According to this view, technologies differences across firms arise as a natural by-product of strategic choices, like in Salant and Shaffer (1999), and more recently in Krysiak (2008), where the policy implications of asymmetric choices are emphasized. ${ }^{2}$ This approach does not deal with the issue of why obsolete technologies persist, but rather focuses on asymmetric equilibrium choices. To be precise, cost and technology asymmetries of two main types have been analyzed: the first is with higher versus lower average (and total) costs, namely superior versus inferior technologies (e.g. Bester and Petrakis, 1996, and more recently Amir et al., 2011); the second is with cost functions that cross at a single point, so that one technology is more efficient at low and the other at high output levels. This second type of asymmetry is the only one considered in this paper. Several works in various context adopt a similar approach (Mills and Smith, 1996; Wauthy and Zenou, 2000; Elberfeld, 2003; Götz, 2005; Hansen and Nielsen, 2010). It has also been argued that cost asymmetries are used by firms to strategically manipulate their cost structures (Van Long and Soubeyran, 2001) or help less efficient rivals in order to achieve higher profits (Ishida et al., 2011). Also, the choice of flexible versus rigid technologies in terms of adaptability to demand has been analyzed in Goyal and Netessine (2007). Bustos (2011) adopts a definition of the technology set that is similar to ours, in a model of international trade where however strategic interaction is absent.

With respect to the literature, we introduce a (endogenous) relation between the technology choice and the position in the product space; we also aim at uncovering the long-run policy implications of technology choices. We set our main focus on the following issues: (i) how and if the technology choice depends upon the position of a firm in the product space; (ii) what is the impact of exogenous changes, and more specifically of shocks on either fixed

\footnotetext{
${ }^{1}$ We do not refer here to the rich literature in Management which also offers important contributions, see for instance McEvily and Zaheer (1999)

${ }^{2}$ On similar grounds, Février and Linnemer (2004) study the effects of exogenous asymmetric shifts in marginal costs in Cournot oligopolies.
} 
or marginal costs, on equilibrium technologies and prices; (iii) how are technology choices affected by policy interventions. Regarding the first question, we distinguish two types of firms: a "central" one and two "peripheral" or "marginal" firms. These roles naturally follow by placing the three firms respectively at the center and at the two extremes of a Hotelling "linear city" model. The position of a firm will affect its equilibrium market share, with marginal firms normally enjoying a lower share. Since firms are competing in prices and since prices are strategic complements, a higher marginal cost of rivals benefits a firm in terms of market share and profits and in turn affects its decision about which technology to choose. This interdependence is at the heart of our analysis.

The choice set for each firm in our model is composed of two alternatives: a technology exhibiting constant returns to scale (CRS) and one with increasing returns to scale (IRS). Technologies are chosen at the first stage of the game and at the second stage price competition is resolved. The IRS technology implies a positive fixed cost and no marginal cost, while the CRS displays a zero fixed cost and a positive marginal cost - more generally the lower marginal or fixed cost could be positive instead of zero as far as the differences are preserved. The central firm is naturally endowed with a larger market share if technologies are the same for all firms and has a higher convenience to adopt a technology exhibiting increasing returns to scale. Indeed there is no equilibrium in which the central firm adopts the CRS except for the equilibrium where all firms do - obviously when the fixed cost in the IRS is too high with respect to the marginal cost in the CRS. It is also interesting that a peripheral firm only adopts the IRS technology if the central firm also does so. In the range of possible asymmetric equilibria, interestingly, there is a parameter region where the two peripheral firms choose the same technology (a CRS) and a region where they choose different technologies. ${ }^{3}$

The increase in fixed costs in switching to the IRS technology takes an important role and the usual irrelevance of fixed costs in determining prices in oligopoly does not hold in this context. An exogenous change in fixed costs of the IRS technology may lead to changes in the technology configuration, with consequences on the output prices, contrary to what happens in short-run models where technologies are given exogenously. For example a decrease in the fixed cost associated to the IRS technology may lead to additional firm(s) adopting it, with a lower price for all firms, due to prices being strategic complements. For similar reasons, an increase in the marginal cost implied by the CRS technology may lead to a change favouring the IRS one and a reduction in prices. The degree of competitiveness in the market, as indexed by the reciprocal of the transportation cost, also affects the equilibrium outcome, with an increase in competitiveness making it more likely that firms choose asymmetric technologies rather than symmetric ones.

\footnotetext{
${ }^{3}$ This confirms the intuition that firms with higher variable costs than those of the competitors are often associated with niche-products, although we do not venture here in giving a cause-effect ordering to the association; this would imply a full analysis of the location choice game.
} 
The price distribution will be with symmetric prices only if technologies are symmetric, otherwise the prices will differ across firms.

It is then natural to analyze the effects of some policy actions by the Government. A price cap, limiting the possibility to adjust the price upward may deter the switch from an IRS to a CRS technology by one firm, when this would be done in the absence of a cap. This is a further limitation to the profit seeking behavior by regulated firms, beyond the simple impediment to raise price. Taxes and subsidies also may change the technology choices as far as they change one or the other cost (fixed or marginal). As for non-discriminatory taxes, namely affecting all firms independent of their technology, we find that while a nondiscriminatory unit tax never changes the equilibrium choices, a nondiscriminatory ad-valorem tax does so in some regions of the parameter space. Discriminatory taxes, aimed to discourage the adoption of a specific technology and that therefore are applied only to firms adopting it, may change the equilibrium configuration. Finally, a tax on a variable input works in the same way as a discriminatory tax and increases the attractiveness of the IRS technology; hence a tax, say, on a polluting variable input reduces the output of the firms that are using this input more intensively and can trigger the adoption of the alternative IRS technology.

The plan of the paper is as follows. In Section 2 we describe our model. In Section 3 we first find the possible equilibrium configurations as functions of the cost parameters, showing that not all possible configurations may arise as Nash equilibria, then we analyze some consequences of changes in costs that may alter the equilibrium configurations, as well as the effect of an increase in the competitiveness of the market (a reduction in product differentiation). In Sections 4, 5, 6 and 7 we the analyze the effects of some frequently used policies like a price cap, a unit tax or subsidy on output, an ad-valorem tax, a tax or a subsidy on an input. Finally, in the concluding Section 8 we summarize our findings and add some comments.

\section{Model}

We consider a Hotelling "linear city" with uniform distribution of a mass $M=1$ of consumers over the interval $[0,1]$ which represents the product space. We assume linear transportation costs $t\left(x-x_{i}\right)$ where $x_{i}$ is the location of firm $i$ in the unit interval and $x$ the location of a consumer. Consumers are uniformly distributed. There are three firms with exogenous locations $x_{1}=0, x_{2}=1 / 2, x_{3}=1$. The extreme location firms take the role of "peripheral". 4

Two cost functions are available to the firms. The first one is characterized by very low (zero) fixed costs and positive marginal costs, thereby displaying constant returns-to-scale

\footnotetext{
${ }^{4}$ Endogenous locations in a 3-firms Hotelling model are not obvious and are studied in De Palma et al. (1987).
} 
$(\mathrm{CRS})$ and is such that $C_{c}\left(q_{i}\right)=c q_{i}, c>0$. The second has positive fixed costs and very low (zero) marginal costs, thereby displaying increasing returns-to-scale (IRS) and is such that $C_{f}\left(q_{i}\right)=f, f>0$, the two cost functions cross at $q=f / c$.

Since the indifferent consumers are $x_{12}=\frac{1}{4}+\frac{p_{2}-p_{1}}{2 t}$ (indifferent between firms 1 and 2) and $x_{23}=\frac{3}{4}-\frac{p_{2}-p_{3}}{2 t}$ (indifferent between firms 2 and 3 ), the demand system is given by the following equations:

$$
\begin{gathered}
q_{1}\left(p_{1}, p_{2}\right)=1 / 4+\left(p_{2}-p_{1}\right) /(2 t) \\
q_{2}\left(p_{1}, p_{2}, p_{3}\right)=1 / 2+\left(p_{1}+p_{3}-2 p_{2}\right) /(2 t) \\
q_{3}\left(p_{2}, p_{3}\right)=1 / 4+\left(p_{2}-p_{3}\right) /(2 t)
\end{gathered}
$$

It is apparent that the demand to the central firm at symmetric prices, has a higher intercept and a higher own price elasticity than that of those for the peripheral firms, while the cross elasticities are symmetric.

Given the technologies adopted in the industry, each firm $i \in\{1,2,3\}$ maximizes its profit $\pi_{i}\left(p_{i}, \mathbf{p}_{-\mathbf{i}}, \tau\right)=q_{i}\left(p_{i}, \mathbf{p}_{-i}\right) p_{i}-C_{\tau}\left(q_{i}\right)$, where $q_{i}$ is as in (1), $\tau \in\{c, f\}$ and $\mathbf{p}_{-\mathbf{i}}$ is the (vector of) the rivals' prices.

Then, the best reply functions are implicitly characterized by the following condition for $i=1,2,3$ (arguments omitted)

$$
p_{i}=C_{\tau}^{\prime} q_{i}^{\prime}-\frac{q_{i}}{q_{i}^{\prime}}
$$

If firms had different marginal costs their best replies would write as

$$
\begin{aligned}
& p_{i}=\left(t+2 c_{i}\right) / 4+p_{2} / 2, \text { for } i \in\{1,3\} \\
& p_{2}=\left(t+2 c_{2}\right) / 4+\left(p_{1}+p_{3}\right) / 2 .
\end{aligned}
$$

\section{$3 \quad$ Equilibrium analysis}

The firms play a two-stage game, at the first stage they simultaneously choose which technology to adopt, at the second they simultaneously set their prices. Let the triplet 123 represent the technology choices of firms 1,2,3 respectively, so that, for instance, $c f c$ means that firms 1 and 3 select the CRS technology and firm 2 selects the IRS one. There are eight possible technology configurations, namely $f f f, f f c, c f f, f c f, f c c, c c f, c f c$ and $c c c$. Clearly, configurations $f f c$ and $c f f$ are equivalent to each other up to a permutation in the labels of the marginal firms, and so are $c c f$ and $f c c$. Hence we have in total only six possible non equivalent configurations that are to be analyzed, namely $f f f, f f c, f c f, f c c, c f c$ and $c c c$. 


\subsection{Price stage}

The firms set their prices simultaneously; hereafter we report the optimal prices, and the relative quantities sold and profits reaped by the firms according to the technologies chosen at the first stage.

\section{(i) Symmetric Configuration $f f f$}

Simultaneously solving the system defined by (2), with $\tau=f$ for $i=1,2,3$ we get symmetric prices,

$$
p_{i}^{f f f}=t / 2
$$

which lead to quantities $q_{1}^{f f f}=q_{3}^{f f f}=1 / 4$ and $q_{2}^{f f f}=1 / 2$, with profits

$$
\pi_{1}^{f f f}=\pi_{3}^{f f f}=t / 8-f, \quad \pi_{2}^{f f f}=t / 4-f .
$$

Remark 1. In configuration $f f f$, as long as $f \leq t / 8$, the profits of all firms are non negative.

\section{(ii) Configuration cff}

Simultaneously solving the system defined by (2), with $\tau=c$ for $i=1$ and $\tau=f$ for $i=2,3$ we get the three prices

$$
p_{1}^{c f f}=(1 / 2)(7 c+6 t) / 6, \quad p_{2}^{c f f}=(c+3 t) / 6, \quad p_{3}^{c f f}=(1 / 2)(c+6 t) / 6,
$$

which lead to quantities $q_{1}^{c f f}=(1 / 4)(6 t-5 c) /(6 t), q_{2}^{c f f}=(c+3 t) /(6 t)$, and $q_{3}^{c f f}=(1 / 4)(c+$ $6 t) /(6 t)$, finally resulting in the following profits,

$$
\pi_{1}^{c f f}=\left(\frac{1}{8}\right) \frac{(6 t-5 c)^{2}}{36 t}, \quad \pi_{2}^{c f f}=\frac{(c+3 t)^{2}}{36 t}-f, \quad \pi_{3}^{c f f}=\left(\frac{1}{8}\right) \frac{(c+6 t)^{2}}{36 t}-f .
$$

Remark 2. In configuration $c f f$, the quantity produced by firm 1 is non negative as long as $c \leq \frac{6}{5} t$, the profits firms 2 and 3 are non negative as long as $f \leq\left(\frac{1}{8 t}\right)\left(\frac{c+6 t}{6}\right)^{2}$.

It is immediate to observe that, with an appropriate change of the indices, the outcomes under this configuration are equivalent to those of the configuration $f f c$.

\section{(iii) Configuration $c f c$}

Simultaneously solving the system defined by (2), with $\tau=c$ for $i=1,3$ and $\tau=f$ for $i=2$, obtains symmetric prices for firms 1 and 3 and a lower price for firm 2 with:

$$
p_{1}^{c f c}=p_{3}^{c f c}=\frac{3 t-2 c}{6}+c, \quad p_{2}^{c f c}=\frac{3 t+2 c}{6},
$$


which lead to the quantities $q_{1}^{c f c}=q_{3}^{c f c}=\left(\frac{1}{2}\right) \frac{3 t-2 c}{6 t}$, and $q_{2}^{c f c}=\frac{3 t+2 c}{6 t}$, resulting in the following profits

$$
\pi_{1}^{c f c}=\pi_{3}^{c f c}=\left(\frac{1}{2}\right) \frac{(3 t-2 c)^{2}}{36 t}, \quad \pi_{2}^{c f c}=\frac{(3 t+2 c)^{2}}{36 t}-f
$$

Remark 3. In configuration $c f c$, the quantities produced by firms 1 and 3 are non negative as long as $c \leq \frac{3}{2} t$, the profits firm 2 are non negative as long as $f \leq \frac{1}{t}\left[\frac{3 t+2 c}{6}\right]^{2}$.

\section{(iv) Configuration $f c c$}

The simultaneous solution of the system defined by equations (2), with $\tau=f$ for $i=1$ and $\tau=c$ for $i=2,3$ returns

$$
p_{1}^{f c c}=(1 / 2)(5 c+6 t) / 6, \quad p_{2}^{f c c}=(3 t-c) / 6+c, \quad p_{3}^{f c c}=(1 / 2)(6 t-c) / 6+c,
$$

leading to the quantities $q_{1}^{f c c}=(1 / 4)[(5 c+6 t) /(6 t)], q_{2}^{f c c}=(3 t-c) /(6 t)$, and $q_{3}^{f c c}=$ $(1 / 4)[(6 t-c) /(6 t)]$, resulting in the following profits:

$$
\pi_{1}^{f c c}=\left(\frac{1}{8}\right) \frac{(5 c+6 t)^{2}}{36 t}-f, \quad \pi_{2}^{f c c}=\frac{(3 t-c)^{2}}{36 t}, \quad q_{3}^{f c c}=\left(\frac{1}{8}\right) \frac{(6 t-c)^{2}}{36 t} .
$$

Remark 4. In configuration $\mathrm{fcc}$, the quantities produced by all firm 1 is always positive, that of firm 2 is positive for $c<3$ t and that of firm 3 is positive for $c<6$. The profits firm 1 are non negative as long as $f \leq \frac{1}{8 t}\left(\frac{5 c+6 t}{6}\right)^{2}$.

With an appropriate change of the indices, the outcomes under this configuration are equivalent to those of the configuration $c c f$.

\section{(v) Configuration $f c f$}

Proceeding as in the previous cases, with $\tau=f$ for $i=1,3$ and $\tau=c$ for $i=2$ in the system defined by (2), we obtain symmetric prices for firms 1 and 3 and a higher price for firm 2, with

$$
p_{1}^{f c f}=p_{3}^{f c f}=(2 c+3 t) / 6, \quad p_{2}^{f c f}=\frac{1}{6}(4 c+3 t),
$$

the relative quantities are $q_{1}^{f c f}=q_{3}^{f c f}=(2 c+3 t) /(12 t), \quad q_{2}^{f c f}=(3 t-2 c)(6 t)$, and the profits are

$$
\pi_{1}^{f c f}=\pi_{3}^{f c f}=\left(\frac{1}{2}\right) \frac{(2 c+3 t)^{2}}{36 t}-f, \quad \pi_{2}^{f c f}=\frac{(3 t-2 c)^{2}}{36 t}
$$

Remark 5. In configuration $f c f$, the quantity produced by firm 2 is positive as long as $c<\frac{3 t}{2}$ and the profits of firms 1 and 3 are non negative as long as $f \leq \frac{(2 c+3 t)^{2}}{72 t}$. 


\section{(vi) Symmetric Configuration ccc}

Last, we consider the case where, in (2), $\tau=c$ for $i=1,2,3$. The optimal prices are symmetric,

$$
p_{i}^{c c c}=c+t / 2
$$

the relative quantities are $q_{1}^{c c c}=q_{3}^{c c c}=1 / 4$, and $q_{2}^{c c c}=1 / 2$, and the profits are

$$
\pi_{1}^{c c c}=\pi_{3}^{c c c}=\frac{t}{8}, \text { while } \pi_{2}^{c c c}=t / 4
$$

In configuration $c c c$ quantities and profits are always positive.

\subsection{Technology choice stage}

Firms simultaneously and costlessly adopt their technologies. In the following, we shall assume

Assumption 1 (A.1). $c<(6 / 5) t$ and $f<t(1 / 8)$.

Under the foregoing Assumption, all the possible technological candidate-equilibrium configurations the quantities and profits of the firms are non-negative. Later in this Section we will discuss how relaxing this assumption affects the equilibrium configurations. In Appendix A we prove the following

Proposition 1. Under A.1, let $F^{I} \equiv \frac{5 c}{24 t}\left(t-\frac{5 c}{12}\right), F^{I I} \equiv \frac{5 c}{24 t}\left(t-\frac{c}{4}\right)$ and $F^{I I I} \equiv \frac{c}{3 t}\left(t+\frac{c}{3}\right)$, where the ranking $F^{I}<F^{I I}<F^{I I I}$ holds, then neither $f c c$ (and ccf) nor fcf can be equilibrium configurations, and

(i) for $0<f<F^{I}$ a unique equilibrium exists, at which the technology choices are $f f f$,

(ii) for $F^{I}<f<F^{I I}$ a unique (up to a permutation of the labels of the marginal firms) equilibrium exists, at which the technology choices are $\operatorname{cff}($ or $f f c)$,

(iii) for $F^{I I}<f<F^{I I I}$ a unique equilibrium exists, at which the technology choices are cfc,

(iv) for $F^{I I I}<f$ a unique equilibrium exists, at which the technology choices are ccc.

It is useful to observe that under A.1 neither $f c c$ (and ccf) nor $f c f$ can be equilibrium configurations. In fact, in the first case, either firm 1 wants to deviate (when $\mathrm{f}$ is "large" relative to $c$ ) or firm 2 wants to deviate (when $f$ is "small" relative to $c$ ). In the second case a similar reasoning applies, but in this case, both marginal firms want to deviate to technology c. Indeed a Corollary can be stated to highlight the differences between the central and peripheral firms. 
Corollary 1. If the CRS technology is adopted by a single firm this is a peripheral one. If the IRS technology is adopted by a single firm this is the central one.

To understand Proposition 1, start with a low level of $f$ relative to $c$, like case (i) in the Proposition. As is intuitive, all firms select the IRS technology, which allows to save on variable costs. For larger values of $f$, however, the CRS technology becomes more attractive and, eventually, only one of the marginal firms switches to this technology (case (ii)). It is interesting to observe that in spite of their symmetry with respect to the central location, the marginal firms make different choices in this region. Furthermore, the firm that switches to the CRS technology increases its marginal cost and therefore optimal price. Because of the strategic interaction the prices of firms 2 and 3 increase too. This price increase is beneficial to 2 and 3, leading to an increase in their profits, both because they serve larger market shares and enjoy increased mark-ups than they did before the change. The region for this situation is with $F^{\prime}<F<\tilde{F} \equiv \frac{c}{3}-\frac{c^{2}}{9 t}$. By contrast, in this region the profits of the switching firm are decreased.

As $f$ increases further (falling in case (iii) or $c f c$ ), the second peripheral firm also adopts the CRS technology, while the central firm sticks to the IRS one. This is explained by the larger market share of firm 2, due to its central location, which implies that this firm has a "comparative advantage" in adopting the IRS technology. Finally, when $f$ is very large, also this firm abandons the IRS technology leading to the configuration ccc. Figure 1 graphically represents the regions in Proposition 1, in particular Panel (1a) plots these regions as function of the marginal (and average) cost $c$, for given $t$ and Panel (1b) as a function of $t$ for given $c$.

The foregoing Proposition has some immediate consequences stated in the following result.

Corollary 2. Fixed costs are consequential regarding the (long-run) price level in the industry. Any increase (decrease) in $f$ inducing a change in the technology equilibrium configuration yields to a generalized though indirect effect in the price level.

Proof. Directly follows from the comparison of the prices set by firms in the different regions identified in Proposition 1.

As an example of the price effect in the Corollary, suppose that the cost parameters initially lead to the configuration $c c c$. In this situation the price is given by (13) for all firms, namely $p=c+t / 2$. Suppose now that a decrease in $f$ leads to the configuration $c f c$ (because $f$ decreases from $F^{I I I}<f$ to $F^{I I}<f<F^{I I I}$. The highest price is the common price of the peripheral firms in (7), which is lower than the common price $c+t / 2$ prevailing before the change of technology by firm 2 . Hence in this case the decrease in $f$ brings forth a generalized (namely for each individual firm) price decrease. This need not be always true, but more generally one can state the following. 


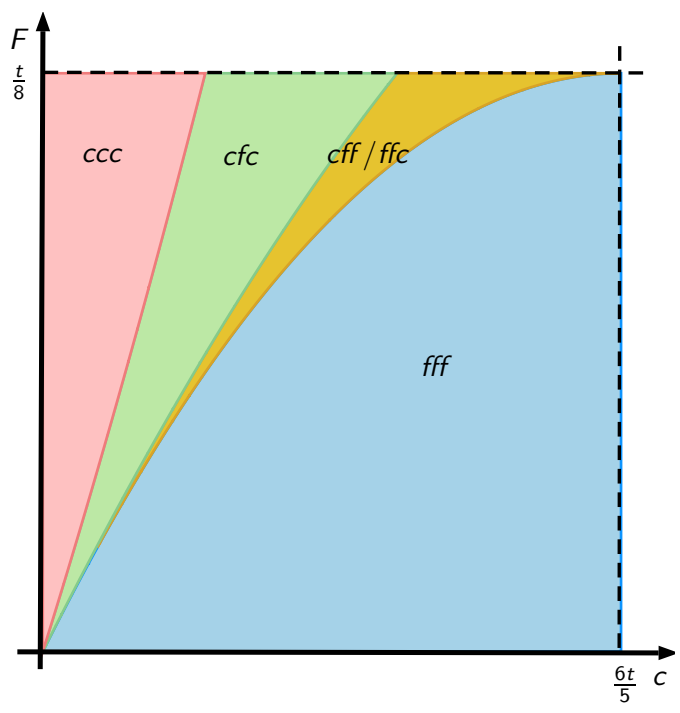

(a) $t=1$

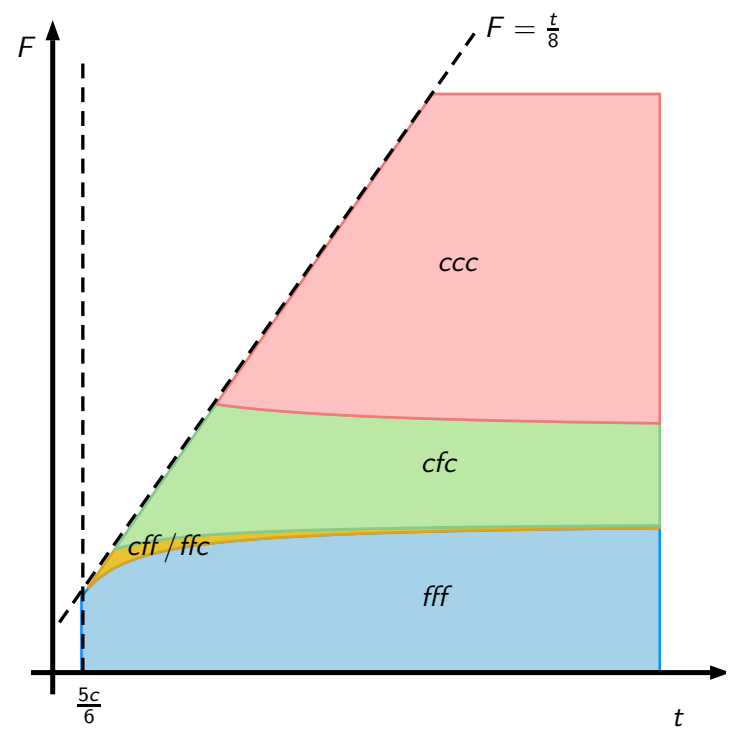

(b) $c=1$

Figure 1: Equilibrium technology choice.

Proposition 2. As $f$ decreases from a level such that $f>F^{I I I}$ continuously, then at each change in configuration there corresponds a lowering of the average price paid by the consumers.

Proof. Let $\min \left\{p^{c f c}\right\}$ (respectively $\max \left\{p^{c f c}\right\}$ ) denote the lowest (respectively, the highest) price when the equilibrium configuration is $c f c$, and use similar notation for the other configurations. Then starting with $f>F^{I I}$, and letting $f$ decrease continuously the equilibrium changes from $c c c$ to $c f c$, then from $c f c$ to $c f f$, and finally from $c f f$ to $f f f$. Since the relation $\min \left\{p^{c c c}\right\}>\max \left\{p^{c f c}\right\}$ holds and since $\min \left\{p^{c f f}\right\}>\max \{p(f f f)\}$ holds then the weighted average price at which goods are sold decreases in the first and third change in configuration triggered by a continuous decrease in $f$. The relation $\min \left\{p^{c f c}\right\}=p_{2}^{c f c}>\max \left\{p^{c f f}\right\}=p_{2}^{c f c}$ however does not hold true and one has to check that the average price decreases in the change. Let $\bar{p}^{c f c}=p_{1}^{c f c}\left(q_{1}+q_{3}\right)+p_{2}^{c f c} q_{2}^{c f c}$ and $\bar{p}^{c f f}=p_{1}^{c f f} q_{1}^{c f f}+p_{2}^{c f f} q_{2}^{c f f}+p_{3}^{c f f} q_{3}^{c f f}$ Then it is a mater of computation to show that $\bar{p}^{c f c}-\bar{p}^{c f f}=\frac{1}{48} \frac{c}{t}(12 t-c)$ which is positive for the range of $c, t$ values where $c<6 t / 5$.

According to Proposition 2, holding $c$ constant, the price of any firm is a function $p_{i}(f ; c)$ of $f$, with the graph of a step function displaying positive upward "jumps" at the critical points $F^{I}, F^{I I}, F^{I I}$, the right-most interval taking value $p_{i}=(c+t) / 2$, for $i=1,2,3$. Starting from the left, the first interval corresponds to configuration $f f f$, the second to $c f f$, the third to $c f c$, the fourth to $c c c$. Panel (2a) of Figure 2 provides a diagrammatical representation of this point with reference to $p_{2}$. 


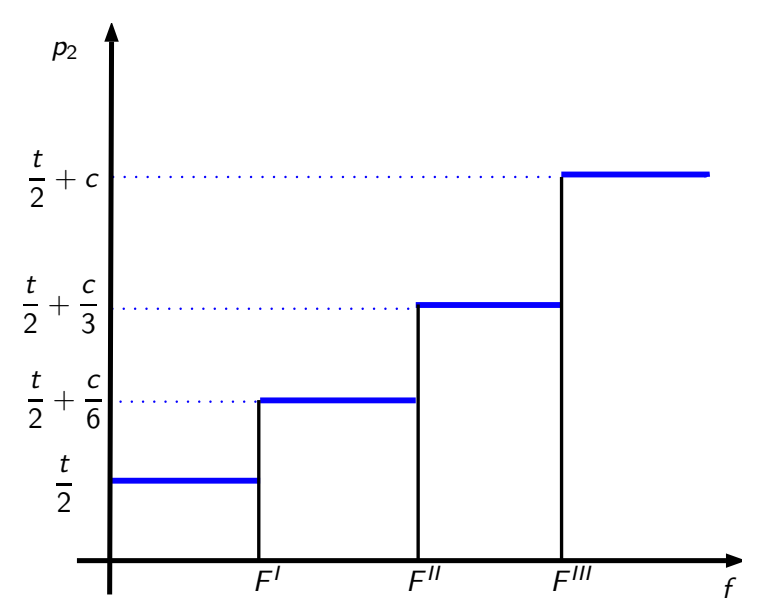

(a) $p_{2}(f ; c)$

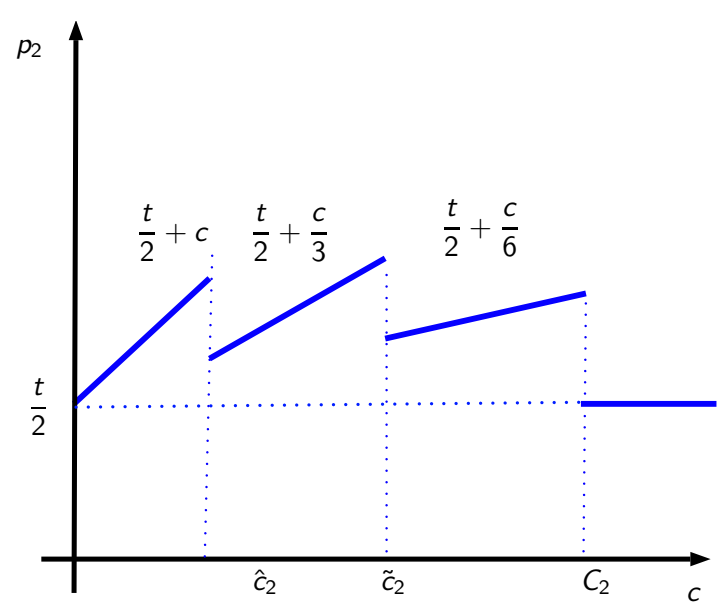

(b) $p_{2}(c ; f)$

Figure 2: $p_{2}$ as a function of the parameters.

Any decrease in the fixed production cost makes the IRS technology relatively more attractive than the CRS one and eventually, when the decrease is such that one of the region boundaries identified in Proposition 1 is crossed, one firm abandons the CRS and switches to the IRS technology. This has an immediate effect on the cost structure of the switching firm, which experiences a decrease in the marginal production cost. This causes the price reaction function of this firm to shift down and to the left. The rival firms react to the price decrease by setting lower prices as well since prices are strategic complements - no change in their reaction function occurs. Conversely, but for analogous reasons, an increase in a fixed cost, which is usually assumed to be price-neutral, can generate an increase in the price level.

A mirror-image reasoning applies for an increase in the marginal production cost. In fact it is easy to observe that the region boundaries $F^{I}, F^{I I}$ and $F^{I I I}$ are all increasing in $c$ for $c<\frac{6 t}{5}$. This entails that, for given $f$, an increase in the marginal production cost $c$ just above a threshold point can lead to a price reduction instead of a price increase. The relation between marginal cost in the CRS and prices are non monotonic. To see this consider a configuration where at least one firm is operating the CRS technology. For given $f$, assume now that $c$ increases. Clearly, if the shift is large enough, the IRS technology becomes a more profitable choice for at least one firm that before the change was adopting the CRS technology. For this firm the switch would imply a fall in its marginal cost (here a fall to zero) and a price decrease by this firm, followed by a strategic price cut by the rivals. Hence we can state the following result.

Corollary 3. The relations $p_{i}(c ; f)$ linking the marginal cost, $c$, in the CRS and the equilibrium prices of firms $i=1,2,3$ for a given value of $f$, are non monotonic; furthermore, in 
some intervals of the cost parameter $c$, an increase (decrease) in c triggering a change in the technology equilibrium configuration, leads to a generalized reverse change in the prices of all firms.

The general picture of the dependence of prices on marginal cost in the CRS is made more clear by considering that, holding $f$ constant, as $c$ is let to vary the price of any one firm is a function $p_{i}(c ; f)$, that can be graphed as a piecewise linear function, displaying two flat portions at the extreme left and right intervals, and a saw-like shape in the middle intervals, with the intervals defining different technology configurations. Starting from the $c=0$, the first interval corresponds to configuration $c c c$, the second to $c f c$, the third to $c f f$, the fourth to $f f f$. At each threshold level for $c$ between one and the next interval, a discontinuity in $p_{i}(c ; f)$ occurs with a vertical drop in the downward direction. For instance, analyzing the relation for firm 2, let $\hat{c}_{2}=\frac{3 t}{2}\left(\sqrt{\frac{4 f+t}{t}}-1\right)$ be the critical level where $\pi_{2}^{c c c}=\pi_{2}^{c f c}$ so that when $c$ raises above $\hat{c}_{2}$ firm 2 switches from the CRS to the IRS technology. Let $\tilde{c}_{2}$ the one where $\pi_{3}^{c f c}=\pi_{3}^{c f f}$, so that when $c$ raises above $\tilde{c}_{2}$ firm 3 switches from the CRS to the IRS technology, $\hat{c}_{2}<\tilde{c}_{2}$. The graph of $p_{2}(c ; f)$, displays a linearly increasing portion over the interval $[0, \hat{c})$ with $p_{2}=p_{2}^{c c c}=t / 2+c$ and a linearly increasing portion over the interval $(\hat{c}, \tilde{c})$ with $p_{2}=p_{2}^{c f c}=t / 2+c / 3$, however it displays a downward vertical shift (of size $(2 / 3) \hat{c}_{2}$ ), at the point $c=\hat{c}_{2} \cdot{ }^{5}$ Panel $2 \mathrm{~b}$ of Figure 2 provides a diagrammatical representation.

Our last result in the equilibrium analysis concerns the role of $t$, the transportation cost, which translates into the substitutability between products. A lower $t$ increases substitutability and hence the degree of competition in a Bertrand framework. The size of the regions for $f$ that lead to $(f f f)$ and to $(c c c)$ decrease as $t$ decreases; by contrast the size of region for $(f f c)$ or $(c f f)$ and that for $(c f c)$ both increase. Hence we can state also the following result.

Corollary 4. Any increase in the competitiveness of the market (a decrease in $t$ ) decreases the likelihood that the final configuration will be symmetric.

Before proceeding, for the sake of completeness we explore the implications for the equilibrium technological configuration of the restriction in A.1, namely $f<\frac{t}{8}$ and $c<\frac{6 t}{5}$, which are diagrammatically reported in Figure 3. As pointed out above, these two conditions insure that at all candidate equilibrium technological configurations, the quantities and profits of all firms are non-negative. Therefore, relaxing A.1 implies that the non-negativity of profits is no longer guaranteed for the firms adopting the IRS technology, because the fixed production

\footnotetext{
${ }^{5}$ At this value there is a second downward drop (of size $(1 / 6) \tilde{c}_{2}$ ). Then the price increases linearly over the interval $\left(\tilde{c}_{2}, C_{2}\right)$, where at $C_{2}$ the profit equality $\pi_{1}^{c f f}=\pi_{1}^{f f f}$ triggers the "last" firm 1 to also embrace the technology with IRS and the price of firm 2 drops to the constant value $p_{2}=t / 2$. At $c=C_{2}$ therefore there is a third and final drop in $p_{2}$ of size $\frac{1}{6} C_{2}$. Over the interval $\left(\hat{c}_{2}, C_{2}\right)$ the price of firm 2 takes a sawlike shape, whence the non-monotonicity . A similar pattern-with different intermediate values and different critical values-is displayed by $p_{1}$ and $p_{3}$.
} 


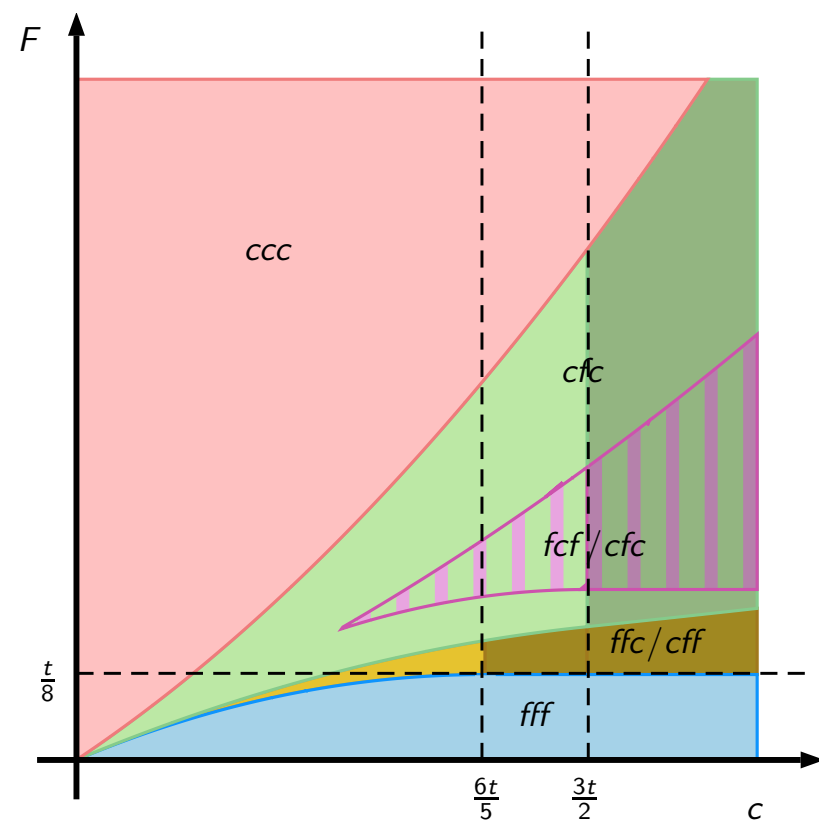

Figure 3: Technology equilibrium configurations with unrestricted parameter space.

cost may exceed the revenues of the firm. Similarly, the positivity of output (and thereby of profits) of the firms running the CRS technology is no longer guaranteed either. Keeping this in mind, if $f>\frac{t}{8}$, the profits of the firms under technological configuration $f f f$ become negative, implying that each firm has an incentive to switch to the CRS technology, ultimately entailing that $f f f$ can no longer be part of a SPNE, for any level of $c .{ }^{6}$ On the other hand, in the case $c>\frac{6 t}{5}$ the optimal quantity produced by a firm running the CRS technology equals zero under some technological configurations. In particular, when $c \in\left[\frac{6 t}{5}, \frac{3 t}{2}\right]$, this happens in configurations $c f f$ and $f f c$, and when $c>\frac{3 t}{2}$ this happens in configuration $c f c$ too. ${ }^{7}$ In the remainder of the paper, we will stick to A.1.

\section{Price-cap regulation}

In the present framework, price-cap regulations may hamper upward price adjustments that follow the adoption of the CRS technology by one or more firms. Therefore they may dis-

\footnotetext{
${ }^{6}$ To this regard, it is instructive to observe that the threshold $F^{I}$ equals $\frac{t}{8}$ for $c=\frac{6 t}{5}$.

${ }^{7} \mathrm{~A}$ second consequence of relaxing the restrictions on the parameters is that for $c>\frac{6 t}{5}$ equilibrium multiplicity appears in a sub-region of the space $F^{I I}<f<F^{I I}$. In fact, within this region, $f c f$ is part of a SPNE for $\frac{c}{3}-\frac{c^{2}}{9 t}<f<\frac{5 c}{24}+\frac{5 c^{2}}{96 t}$ and $c>\frac{36 t}{47}$, together with $c f c$ (the green-purple region of Figure 3 (see Appendix A for the derivation of the conditions for the existence of the $f c f$ equilibrium). Notice that, in this "new" equilibrium configuration, the output of the CRS firm is nil for $c>\frac{3 t}{2}$ as well. In Figure 3 the darker regions identify the parameter constellations where the output (and profit) of the firms running the CRS technology are zero at equilibrium. A formal characterization of the technological equilibrium partition of the unrestricted parameter space is available from the authors upon request.
} 
favor this technology. Otherwise stated, when the price cap is fixed below the highest price prevailing under the unregulated equilibrium, the regulated equilibrium can entail a different technology configuration than the unregulated one. In an industry where both technologies do not change often this means that a new equilibrium under regulation will emerge and persist for a medium-long period. In an industry where at least one of the two alternative technologies experience an important shock, the price cap may lead to inability to readjust by some or by all firms.

For instance, assume that the initial cost configuration is $c f c$ and that marginal costs decrease due to changes in labor productivity due to technical progress in the CRT technology, or to decreases in the cost of raw materials. Assume this cost decrease to be important enough to trigger a switch in an unregulated industry from the $c f c$ to the $c c c$ configuration, which would trigger a generalized rise in prices. The firm that has to switch to the CRS technology is the central one. However, if there is a price cap equal to the highest historically inherited price, or anywhere below the unregulated equilibrium price $p^{c c c}$ the technology change may become unprofitable for the central firm.

The profit of the firms under a price cap, represented by the price $p=z$, when they all adopt the CRS technology are $\pi_{i}(z, c c c)=(z-c) \frac{1}{4}$, for $i=1,2,3$.

It is easy to check that, the switch to the CRS technology is not profitable for firm 2 as long as

$$
z<\bar{z} \equiv \frac{(2 c+3 t)^{2}}{18 t}+\frac{3 c}{2}-2 f
$$

It is a matter of calculations to ascertain that $\bar{z} \in\left[p_{1}^{c f c}, p_{1}^{c c c}\right]$, namely the price cap threshold for a profitable technological switch lies in between the unregulated and the maximum inherited price, for $c \in[0, .237 t]$ and $F<c\left(\frac{1}{2}+\frac{c}{9 t}\right)$, or $c \in[.237 t, .337 t]$.

A change from $c f f$ to $c f c$ or from $f f f$ to $f f c$ can be hampered by the same argument. As a general point: a price cap may lead to favor the IRS technology and to alter the equilibrium configuration. Since all the changes where at least one firm switches from the IRS to the CRS technology lead to a price increase, the price cap may play a role in affecting the market outcome even if it is not binding in equilibrium, the relevant equilibrium price being the one that would prevail after the change - the change that however cannot happen.

\section{Unit tax (subsidy) on output}

\subsection{Non-discriminatory unit tax (subsidy)}

With a non-discriminatory unit tax (subsidy) $\theta>0 \quad(<0)$ that affects all firms in the same way, independent of the technology choice, the profits of the firms are $\pi_{i}(\theta)=q_{i}\left(p_{i}, \mathbf{p}_{-i}\right)\left(p_{i}-\right.$ $\theta)-C_{\tau}\left(q_{i}\right)$. It is a matter of easy calculations to ascertain that a unit tax (subsidy) affects all 
the best replies, in all possible configurations, symmetrically by shifting them by a factor $\theta / 2$. This, together with market coverage which implies an inelastic aggregate demand, results in a complete pass through of the tax, which generates a rise in all equilibrium prices, in all configurations, by an amount equal to the $\operatorname{tax}, \theta$. The ultimate consequence is that the candidate-equilibrium profits at each technological configurations do not change relative to the no-tax case. Clearly, this also implies that the equilibrium technological choices under a per-unit tax coincide with those reported in Proposition 1. We summarize this finding in the following

Proposition 3. A nondiscriminatory per-unit tax (or subsidy) on output does not affect the technological choices of the firms.

\subsection{B. Discriminatory unit tax (subsidy)}

The case of a discriminatory tax is also interesting. Assume first that the Government wants to discourage the adoption of the technology using the variable input intensively. In this case it may adopt a unit tax that only applies to the firms using the CRS technology. Obviously this will change the technology choices only if the tax is high enough. As it will be clear from what follows, in our model, such a tax is equivalent to a tax on the variable input, so that we defer its analysis to the following section.

The case where the Government wants to encourage the adoption of the CRS technology is different. In that case the tax should only affect the firms choosing the IRS technology. Starting with the configuration $c f c$, for instance, this discriminatory tax will affect the market structure when the tax is high enough to forestall the adoption by firm 2 to the IRS technology. Similarly, the same effect can be induced by providing a discriminatory subsidy to the firms adopting the CRS technology. One may think, as real life examples, to taxes-subsidies that favor labor intensive technologies. For instance these technologies help preserving jobs, or preserving the environment (like in fisheries), or both. The difference between a tax on the IRS technology and a subsidy on the CRS one is that the first has a negative and the second has a positive effect on consumer surplus, via the effect of equilibrium prices. Obviously a subsidy must be financed. In theory one can define a lump-sum tax on profits of the firms and finance the subsidy, in a way to change the equilibrium technology choice while preserving budget balance for the Government. This is admittedly requiring quite a lot of information on the part of the authorities.

Starting with the configuration $c f c$, the discriminatory $\operatorname{tax} \theta$ on the IRS technology acts as a marginal cost increase from zero to a positive level for firm 2 . The threshold level for

$\theta$ that leads firm 2 to change its technology is defined by rewriting the equilibrium prices as $p_{1}(\theta)=p_{3}(\theta)=\frac{3 t+4 c}{6}+\frac{\theta}{3}$ and $p_{2}(\theta)=\frac{3 t+2 c}{6}+\frac{2 \theta}{3}$. So that $\pi_{2}^{c f c}(\theta)=\frac{1}{36 t}(2 c+3 t-2 \theta)-f$, if 
firm 2 adopts the IRS technology. Then the threshold level of tax on the IRS technology is the solution for $\theta$ of $\frac{1}{36 t}(2 c+3 t-2 \theta)^{2}-f=\frac{t}{4} .^{8}$

Likewise, one can find a threshold level for a subsidy $s$ that triggers a change from, say, $c f c$ to $c c c$. A subsidy is equivalent to a decrease in cost $c$ from $c$ to $c-s$. This does not affect profits in the $c c c$ configuration, which remain equal to $\pi_{2}=t / 4$. However, prices in the $c f c$ configuration under a subsidy to the CRS technology become $p_{1}(s)=p_{2}(s)=\frac{3 t+4(c-s)}{6}$, and

$p_{2}(s)=\frac{3 t+2(c-s)}{6}$. The corresponding profit to firm 2 is $\pi_{2}^{c f c}(s)=\frac{[3 t+2(c-s)]^{2}}{36 t}-f$. Hence the threshold value for $s$ is given by setting $\pi_{2}^{c f c}(s)=t / 4$. This provides the same solution as for $\hat{\tau}$. Hence a discriminatory tax and a discriminatory subsidy have the same magnitude and opposite sign, but the first is enacted upon the IRS technology while the second on the CRS.

Proposition 4. A discriminatory subsidy to favor the CRS technology in the configuration $c c c$, discourages the adoption of the IRS technology and may deliver a decrease in the average price.

Therefore the subsidy eventually grants a double dividend as it achieves the desired technology target and also allows consumers to enjoy a higher surplus. A tax on the IRS does not lower the prices in the $c c c$ configuration and it only achieves the technology target.

\section{Ad-valorem tax}

An ad-valorem tax reduces the profits from both types of technology, because, in this case, the profits accruing to the firms are $\pi_{i}\left(p_{i}, p_{-i}, t, v\right)=q_{i}\left(p_{i}, p_{-i}\right) p_{i}(1-v)-C_{t}\left(q_{i}\right)$, where $v \in[0,1[$ is the tax rate. An analysis analogue to the one of Section 3 can be carried out to obtain the following.

Proposition 5. An ad-valorem tax on output can lead to a change in the technology configuration.

Proof. Assume that $c<\frac{6 t(1-v)}{5}$ and let $F_{v}^{I} \equiv \frac{5 c}{24}-\frac{25 c^{2}}{288 t(1-v)}, F_{v}^{I I} \equiv \frac{5 c}{24}-\frac{5 c^{2}}{96 t(1-v)}$ and $F_{v}^{I I I}=$ $\frac{c^{2}}{9 t(1-v)}+\frac{c}{3}$, with $F_{v}^{I}<F_{v}^{I I}<F_{v}^{I I} \forall t>0, c>0$ and $\left.v \in\right] 0,1\left[\right.$. Then the triplet $\left(F^{I}, F^{I I}, F^{I I I}\right)$ in Proposition 1 is replaced by the triplet $\left(F_{v}^{I}, F_{v}^{I I}, F_{v}^{I I I}\right)$.

The ad-valorem tax affects differently the profitability of each type of technology and, consequently their equilibrium choices. In fact, by analyzing the effect of an increase in $v$ on the threshold values of proposition 5 it is easily obtained that

$$
\begin{gathered}
\frac{\partial F_{v}^{I}}{\partial v}=-\frac{(5 c)^{2}}{288 t(1-v)^{2}}, \quad \frac{\partial F_{v}^{I I}}{\partial v}=-\frac{5 c^{2}}{96 t(1-v)^{2}}, \quad \frac{\partial F_{v}^{I I I}}{\partial v}=\frac{c^{2}}{9 t(1-v)^{2}} . \\
{ }^{8} \text { Namely } \hat{\theta}=c+\frac{3}{2} t\left(1-\sqrt{\frac{4 f}{t}+1}\right) .
\end{gathered}
$$




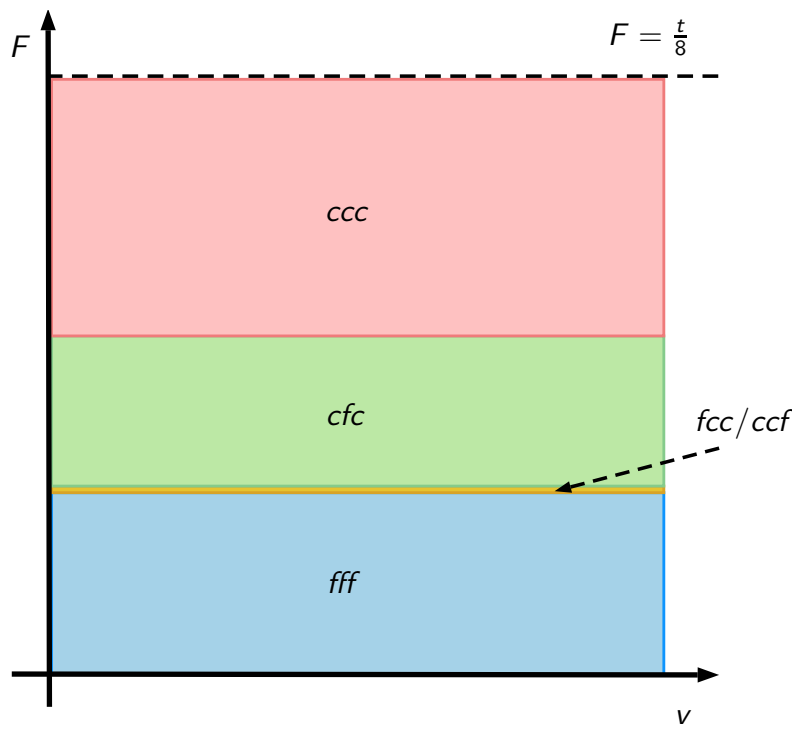

(a) No ad valorem tax

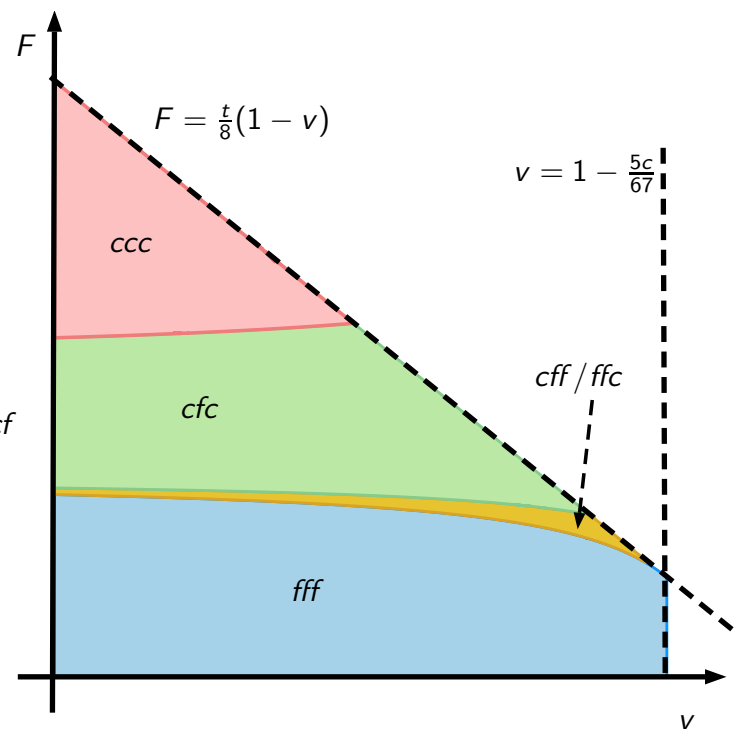

(b) Ad valorem tax

Figure 4: Equilibrium technology choice $(t=3.5, c=.7)$.

It is a matter of easy algebra to ascertain that, $\frac{\partial F_{v}^{I}}{\partial v}<\frac{\partial F_{v}^{I I}}{\partial v}<0$ and that $\frac{\partial F_{v}^{I I}}{\partial v}>$ for all admissible $v$. This implies that the region where "mixed configurations" arise at equilibrium expands relative to the case where there is no tax, Figure 1 plots the threshold values $F^{I}, F^{I I}$ and $F^{I I I}$ (panel (a)) and $F_{v}^{I}, F_{v}^{I I}$ and $F_{v}^{I I I}$ (panel (b)) as a function of the ad valorem tax rate $v$. The following Corollary summarizes these results.

Corollary 5. Any increase in the ad-valorem tax rate $v$ reduces the likelihood that the final technological configuration will be symmetric.

\section{Unit tax on input}

We imagine here that an input like labor, or fuel, can be subject to taxation. This kind of taxation is obviously ubiquitous and has a higher impact on firms adopting the technologies that use more intensively the variable input, like our CRS. It is then clear that the IRS technology becomes relatively more attractive for all firms. The effects on technology adoption are therefore quite straightforward and we shall not pursue the analysis in detail here. The parameter regions where the CRS technology is adopted shrink and those where the IRS is adopted become larger. If one thinks of the taxed input as a polluting one, like fuel, then the tax may be also aiming at reducing emissions. In this case one can compute for the various configurations in which at least one firm is adopting a CRS technology the tax level that leads to a change in technology. As an example, consider the pre-tax equilibrium to be $c c c$. Then 
there is a tax level on input, $\phi^{\prime}$, that causes the central firm 2 to abandon the use of the CRS technology (in our parametrization this also leads to zero utilization of the taxed input). The marginal cost in the CRS technology after the tax $\phi$ per unit of input is introduced becomes $c+\phi$. If all firms adopt the CRS technology the price is $p_{i}=t / 2+c+\phi$ and no change in profits occur, $\pi_{2}^{c c c}=t / 4$. A switch to the IRS technology for firm 2 becomes profitable when $\pi_{2}^{c c c}<\pi_{2}^{c f c}$, or $\frac{t}{4}<\frac{(3 t+2 c+2 \phi)^{2}}{36 t}-f$. The threshold tax triggering the change from $c c c$ to $c f c$ is therefore

$$
\phi^{\prime}=\frac{3}{2}\left(\sqrt{\frac{4 f+t}{t}}-1\right) t-c .
$$

When the central firm operates the switch to the IRS technology, the price it sets is subject to two opposite effects: it is reduced due to the reduction in its own marginal cost and is increased thanks to the increase in the marginal cost of the other two firms. The average price level can therefore go up or down and the policy can eventually be designed so as to deliver a price decrease. Intuitively, when the tax rate on fuel, say, is increased from a preexisting level close to $\phi^{\prime}$ to a level slightly above it, then the average price is going to be lowered as the price complementarity pushes down the prices of the peripheral firms.

It is interesting that the consumption of the taxed input is reduced whether or not a change in technology occurs, so that the aim of the tax is achieved. A change in technology, if it occurs, reinforces the effectiveness of the policy, by inducing a discontinuity in the amount of input in the production function of the switching firm(s).

\section{Conclusion}

In this paper we have analyzed the relation between the relative positioning in a market, with a central versus two peripheral firms, and the choice of technologies. We have in particular discussed the strategic choice of adopting a technology that is more efficient at low output levels or one that is more efficient at high output levels. The equilibrium configurations reveal that the central firm adopts the CRS technology (with high marginal costs and low fixed costs) only if all its rivals do the same. Furthermore, exogenous shocks to technologies, that change ther cost structure, lead to nonobvious changes in the equilibrium prices. Imagine to start with a high fixed cost in the IRS technology and with a low marginal cost in the CRS one, so that all firms adopt this second technology. Let the fixed cost decrease: the central firm is the first one to embrace the IRS technology; then as the fixed cost decreases further only one of the peripheral firms adopts it, and finally all firms do. Hence, fixed costs contribute in shaping the market outcome and determine the price configuration. A decrease in the fixed cost leading to a firm switching from the CRS to the IRS technology, induces a generalized price reduction. This long-run effect contrasts with the short-run irrelevance of fixed costs. 
Then we also find that asymmetric results are more likely as competition intensifies (as the transportation cost is reduced).

The analysis of the equilibrium responses to some widely used policies reveal various interesting points. A price cap may reduce the convenience to adopt a CRS technology, thereby preventing a price increase. A nondiscriminatory unit tax on output has no effect but a nondiscriminatory ad-valorem tax has the same effect as an increase in the degree of competitiveness. A discriminatory tax (subsidy) on output that applies only to firms adopting one of the two technologies can discourage the adoption of the taxed technology and encourage that of the technology which is subsidized. The effects on prices can deliver a double dividend if the policy instrument is a subsidy, with consumers also benefiting due to lower equilibrium prices. Finally a tax on a variable input may induce a change in technology from CRS to IRS,namely to the technology making less use of it) and induce a lower utilization of the taxed input. From the perspective of a taxation on an input that damages the environment, this taxation if properly designed may also grant a double dividend: it decreases the utilization of the undesirable input and it lowers prices to consumers.

We have left several questions for further research: one could analyze the incentives and the means by which a firm can increase the rivals' fixed costs. Note indeed that if a firm switches from the IRS to the CRS technology it creates a positive externality to rivals due to the price complementarity: they will enjoy higher market shares and higher mark-ups after the change. If it were possible, therefore, a firm would like to encourage the adoption of the CRS technology by the rivals, e.g. by making the IRS proprietary, or by raising the rivals' fixed costs if they adopt the IRS (as in Hviid and Olczak, 2016). Finally, if the central firm is a firm producing the input that is used in the CRS technology and selling it to the rivals then it would choose the wholesale price so as to manipulate the technology choice by the rivals, namely so as to strategically avoid a change in technology from the CRS to the IRS.

\section{A Proof of Proposition 1}

(i) To prove the existence of configuration $f f f$ we need to check that neither one peripheral firm nor the central firm have incentives to deviate to the CRS technology.

No deviation by peripheral firm.

If firm 1 unilaterally deviates to the CRS technology it reaps a profit equal to $\pi_{1}^{c f f}$, this is not profitable if

$$
\pi_{1}^{f f f} \geq \pi_{1}^{c f f} \Leftrightarrow f \leq \frac{5 c}{24}-\frac{25 c^{2}}{288 t} .
$$

Clearly, this same condition guarantees that firm 3 does not want to deviate to the CRS technology too. 
No deviation by central firm.

There is no profitable deviation to the CRS technology by the central firm when

$$
\pi_{2}^{f f f} \geq \pi_{1}^{f c f} \Leftrightarrow f \leq \frac{c}{3}-\frac{c^{2}}{9 t}
$$

It is easy to prove that both conditions are fulfilled when $f \leq \frac{5 c}{24}-\frac{25 c^{2}}{288 t} \equiv F^{I}$ and that $F^{I}<\frac{t}{9} \forall c<\frac{6 t}{5}$, which insures the positivity of the SPNE profits.

(ii) Existence of a SPNE with technological configuration $c f f$ or $f f c$ requires the following (here we focus on case $c f f$, which, after an appropriate permutation of the firm labels guarantees existence for configuration $f f c$ ).

No deviation by firm 1 .

This requires that

$$
\pi_{1}^{c f f} \geq \pi_{1}^{f f f} \Leftrightarrow f \geq \frac{5 c}{24}-\frac{25 c^{2}}{288 t}
$$

No deviation by firm 2 .

This requires that

$$
\pi_{2}^{c f f} \geq \pi_{2}^{c c f} \Leftrightarrow f \leq \frac{c}{3}
$$

No deviation by firm 3 .

This requires that

$$
\pi_{3}^{c f f} \geq \pi_{3}^{c f c} \Leftrightarrow f \leq \frac{5 c}{24}-\frac{5 c^{2}}{96 t}
$$

The three above conditions are simultaneously satisfied for $F^{I}=\frac{5 c}{24}-\frac{25 c^{2}}{288 t} \leq f \leq$ $\frac{5 c}{24}-\frac{5 c^{2}}{96 t}=F^{I I}$. It is easy to ascertain that, in this region, the profits of the firms running the IRS technology are positive.

(iii) Existence of an equilibrium with configuration $c f c$ requires what follows.

No deviation by peripheral firm.

For firm 1, this requires that

$$
\pi_{1}^{c f c} \geq \pi_{1}^{f f c} \Leftrightarrow f \geq \frac{5 c}{24}-\frac{5 c^{2}}{96 t}
$$

this same condition insures that firm 3 has no profitable deviation either.

No deviation by central firm.

This requires that

$$
\pi_{2}^{c f c} \geq \pi_{2}^{c c c} \Leftrightarrow f \leq \frac{c}{3}+\frac{c^{2}}{9 t},
$$


these conditions are simultaneously fulfilled when $F^{I I}=\frac{5 c}{24}-\frac{5 c^{2}}{96 t} \leq f \leq \frac{c}{3}+\frac{c^{2}}{9 t}=F^{I I I}$. As before, straightforward calculations prove that the profit of the firm adopting the IRS technology is positive within this parameter space.

(iv) Existence of configuration $c c c$ at equilibrium requires the following.

No deviation by peripheral firm.

$$
\pi_{1}^{c c c} \geq \pi_{1}^{f c c} \Leftrightarrow f \geq \frac{25 c^{2}}{288 t}+\frac{5 c}{24 t}
$$

the same condition guarantees no deviation by firm 3 .

No deviation by central firm.

$$
\pi_{2}^{c c c} \geq \pi_{2}^{c f c} \Leftrightarrow f \geq \frac{c}{3}+\frac{5 c^{2}}{9 t}
$$

The two above conditions are simultaneously satisfied when $f \geq \frac{c}{3}+\frac{5 c^{2}}{9 t}=F^{I I I}$.

To complete the proof of Proposition 1 there remains to demonstrate that no equilibrium exists under the configurations $f c f, c c f$ and $f c c$.

1. Equilibrium under configuration $f c f$ requires that, simultaneously

$$
\pi_{1}^{f c f}=\pi_{3}^{f c f} \geq \pi_{1}^{c c f}=\pi_{3}^{f c c} \Leftrightarrow f \leq \frac{5 c}{24}+\frac{5 c^{2}}{96}, \text { and } \pi_{2}^{f c f} \geq \pi_{2}^{f f f} \Leftrightarrow f \geq \frac{c}{3}-\frac{c^{2}}{9 t} .
$$

It is a matter of simple algebra to ascertain that the two conditions above cannot be simultaneously fulfilled under the assumption $f<\frac{t}{8}$.

2. Equilibrium in configuration $f c c$ requires

$$
\pi_{1}^{f c c} \geq \pi_{1}^{c c c} \Leftrightarrow f \leq \frac{5 c}{24}+\frac{25 c^{2}}{288 t}, \quad \pi_{2}^{f c c} \geq \pi_{2}^{f f c} \Leftrightarrow f \geq \frac{c}{3}, \text { and } \pi_{3}^{f c c} \geq \pi_{3}^{f c f} \Leftrightarrow f \geq \frac{5 c}{24}+\frac{5 c^{2}}{96 t} .
$$

As in the previous case, the tree conditions cannot be simultaneously satisfied for $c<\frac{6 t}{5}$.

\section{References}

Amir, R., Halmenschlager, C., and Jin, J. (2011). "R\&D-induced industry polarization and shake-outs". International Journal of Industrial Organization, 29(4):386-398.

Bester, H. and Petrakis, E. (1996). "Coupons and oligopolistic price discrimination". International Journal of Industrial Organization, 14(2):227-242. 
Boucekkine, R., de la Croix, D., and Licandro, O. (2011). "Vintage capital growth theory: Three breakthroughs". In O. de la Grandville, editor, "Economic Growth and Development", chapter 5, pages 87-116. Emerald Publishing Limited.

Bustos, P. (2011). "Trade liberalization, exports, and technology upgrading: Evidence on the impact of MERCOSUR on Argentinian firms". American Economic Review, 101(1):304-40.

De Palma, A., Ginsburgh, V., and Thisse, J.-F. (1987). "On existence of location equilibria in the 3-firm Hotelling problem". The Journal of Industrial Economics, pages 245-252.

Doms, M., Dunne, T., and Roberts, M. J. (1995). "The role of technology use in the survival and growth of manufacturing plants". International Journal of Industrial Organization, 13(4):523-542.

Dosi, G. (1997). "Opportunities, incentives and the collective patterns of technological change". The Economic Journal, 107(444):1530-1547.

Dunne, T. (1994). "Plant age and technology use in us manufacturing industries". The RAND Journal of Economics, pages 488-499.

Elberfeld, W. (2003). "A note on technology choice, firm heterogeneity and welfare". International Journal of Industrial Organization, 21(4):593-605.

Février, P. and Linnemer, L. (2004). "Idiosyncratic shocks in an asymmetric Cournot oligopoly". International Journal of Industrial Organization, 22(6):835-848.

Götz, G. (2005). "Market size, technology choice, and the existence of free-entry Cournot equilibrium". Journal of Institutional and Theoretical Economics JITE, 161(3):503-521.

Goyal, M. and Netessine, S. (2007). "Strategic technology choice and capacity investment under demand uncertainty". Management Science, 53(2):192-207.

Griliches, Z. (1957). "Hybrid corn: An exploration in the economics of technological change". Econometrica, pages 501-522.

Hansen, J. D. and Nielsen, J. U.-M. (2010). "Market Integration, Choice of Technology, and Welfare". Review of International Economics, 18(2):229-242.

Hviid, M. and Olczak, M. (2016). "Raising rivals' fixed costs". International Journal of the Economics of Business, 23(1):19-36.

Ishida, J., Matsumura, T., and Matsushima, N. (2011). "Market competition, r\&d and firm profits in asymmetric oligopoly". The Journal of Industrial Economics, 59(3):484-505. 
Johansen, L. (1959). "Substitution versus fixed production coefficients in the theory of economic growth: a synthesis". Econometrica, pages 157-176.

Jovanovic, B. (1998). "Vintage capital and inequality". Review of Economic Dynamics, 1(2):497 - 530.

Krysiak, F. C. (2008). "Prices vs. quantities: The effects on technology choice". Journal of Public Economics, 92(5-6):1275-1287.

McEvily, B. and Zaheer, A. (1999). "Bridging ties: A source of firm heterogeneity in competitive capabilities". Strategic Management Journal, 20(12):1133-1156.

Mills, D. E. and Smith, W. (1996). "It pays to be different: Endogenous heterogeneity of firms in an oligopoly". International Journal of Industrial Organization, 14(3):317-329.

Salant, S. W. and Shaffer, G. (1999). "Unequal treatment of identical agents in Cournot equilibrium". American Economic Review, 89(3):585-604.

Van Long, N. and Soubeyran, A. (2001). "Cost Manipulation Games in Oligopoly, with Costs of Manipulating". International Economic Review, 42(2):505-533.

Wauthy, X. and Zenou, Y. (2000). "How the adoption of a new technology is affected by the interaction between labour and product markets". In G. Norman and J.-F. Thisse, editors, "Market Structure and Competition Policy: Game-Theoretic Approaches", chapter 12, pages 271-286. Cambridge University Press. 


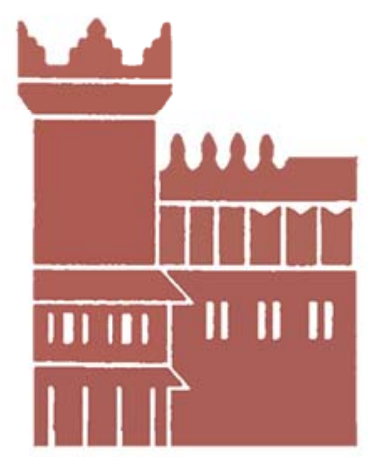

Alma Mater Studiorum - Università di Bologna DEPARTMENT OF ECONOMICS

Strada Maggiore 45

40125 Bologna - Italy

Tel. +39051 2092604

Fax +390512092664

http://www.dse.unibo.it 